\title{
Periodic Solutions for Degenerate Diffusion Equations
}

\section{NoRiko Mizoguchi}

ABSTRACT. In this paper, we are concerned with the existence of nonnegative periodic solutions of

$$
u_{t}=\Delta u^{m}+h(t) f(u) \quad \text { in } \Omega \times \mathbb{R}
$$

with the Dirichlet boundary condition, where $m>1, \Omega$ is a smoothly bounded domain in $\mathbb{R}^{N}$ and $h$ is a given positive periodic function on $\mathbb{R}$. The forcing term in this paper satisfies

$$
\liminf _{\rho \rightarrow \infty} h(t) \frac{f(\rho)}{\rho^{m}}>\lambda_{1} \quad \text { uniformly in } t \in \mathbb{R}
$$

for example, $f(u)=u^{p}$ with $p \geq m$ and $h>\lambda_{1}$. We adapt the Leray-Schauder degree theory in the proof. To do that, we need an a priori estimate for solutions in $L^{\infty}$. Inequalities of Harnack type and the blow up (or scaling) argument play crucial parts.

1. Introduction. We are concerned with the existence of nonnegative solutions of the following problem

$$
\begin{cases}u_{t}=\Delta u^{m}+h(t) f(u) & \text { in } \Omega \times \mathbb{R}, \\ u=0 & \text { on } \partial \Omega \times \mathbb{R}, \\ u(t+T)=u(t) & \text { in } \Omega \times \mathbb{R},\end{cases}
$$

where $m>1, \Omega$ is a smoothly bounded domain in $\mathbb{R}^{N}, h$ is a positive $T$-periodic function on $\mathbb{R}$ and $f$ is a nonnegative function on $\mathbb{R}_{+}$, which is superlinear or asymptotically linear; for example, $f(u)=u^{p}$ with $p \geq m$. Here $\mathbb{R}_{+}$denotes the set of nonnegative real numbers. The problem (1) without periodicity was investigated by many authors (see, e.g., [1], [5], [12], [14], [15]). On the other 
hand, we know few articles on periodic problems. Nokao [13] obtained periodic solutions in a sublinear case. It seems that there are no results when

$$
\liminf _{\rho \rightarrow \infty} h(t) \frac{f(\rho)}{\rho^{m}}>\lambda_{1} \quad \text { uniformly in } t \in \mathbb{R},
$$

where $\lambda_{1}$ is the first eigenvalue of $-\Delta$ with the Dirichlet boundary condition in $\Omega$. In [3] and [4], Esteban showed the existence of positive solutions for a semilinear version of (1) with $\Delta u^{m}$ replaced by $\Delta u$.

Putting $v=u^{m}$ for a nonnegative solution $u$ of (1), $v$ is nonnegative and solves the following quasilinear problem

$$
\begin{cases}v_{t}=m v^{\gamma}\{\Delta v+h(t) \tilde{f}(v)\} & \text { in } \Omega \times \mathbb{R}, \\ v=0 & \text { on } \partial \Omega \times \mathbb{R}, \\ v(t+T)=v(t) & \text { in } \Omega \times \mathbb{R},\end{cases}
$$

where $\gamma=1-1 / m$ and $\tilde{f}(\rho)=f\left(\rho^{1 / m}\right)$. It is clear that the converse implication also holds. Therefore, we shall show the existence of a nonnegative solution of (1) by treating (2) in the present paper. Our purpose is to prove the following two results.

Theorem 1. Let $\Omega$ be convex and $m<p<\infty$ if $N=1$ or $\Omega$ is an open ball in $\mathbb{R}^{2}$ and $m<p<(N m+2) / N$ if $N \geq 2$. Suppose that $f: \mathbb{R}_{+} \rightarrow \mathbb{R}$ is a nonnegative function with $f^{\prime}(\rho) \rho^{1-m} \in C\left(\mathbb{R}_{+}\right)$such that

$$
0<\lim _{\rho \rightarrow \infty} \frac{f(\rho)}{\rho^{p}}<\infty
$$

$$
\limsup _{\rho \rightarrow \infty} \frac{f^{\prime}(\rho)}{\rho^{p-1}}<\infty
$$

and

$$
\lim _{\rho \rightarrow \infty} h(t) \frac{f(\rho)}{\rho^{m}}<\lambda_{1} \quad \text { uniformly in } t \in \mathbb{R} .
$$

If $h: \mathbb{R} \rightarrow \mathbb{R}$ is a T-periodic positive function of class $C^{1}$, then there exists a nonnegative nontrivial weak solution of (1).

Here a weak solution of (1) means a function $u$ such that $u^{m} \in L_{\text {loc }}^{2}\left(\mathbb{R} ; H_{0}^{1}(\Omega)\right) \cap$ $L^{\infty}(\Omega \times \mathbb{R}),\left(u^{m}\right)_{t} \in L_{\text {loc }}^{2}\left(\mathbb{R} ; L^{2}(\Omega)\right), u^{2 m-1} \in C\left(\mathbb{R} ; L^{p}(\Omega)\right)$ for all $1 \leq p<\infty$, $u(t+T)=u(t)$ a.e. in $\Omega$ for all $t \in \mathbb{R}$ and

$$
\iint\left\{u \varphi_{t}-\nabla u^{m} \nabla \varphi+h(t) f(u) \varphi\right\} d x d t=0
$$

for each $\varphi \in C_{0}^{\infty}(\Omega \times(0, T))$. 
Theorem 2. Assume that $f: \mathbb{R}_{+} \rightarrow \mathbb{R}$ is a nonnegative function with $f^{\prime}(\rho) \rho^{1-m} \in C\left(\mathbb{R}_{+}\right)$satisfying

$$
\lambda_{1}<\liminf _{\rho \rightarrow \infty} h(t) \frac{f(\rho)}{\rho^{m}} \leq \limsup _{\rho \rightarrow \infty} h(t) \frac{f(\rho)}{\rho^{m}}<\infty \text { uniformly in } t \in \mathbb{R}
$$

and the assumptions (4) with $p=m$ and (5) in Theorem 1 . If $h: \mathbb{R} \rightarrow \mathbb{R}$ is a T-periodic positive function of class $C^{1}$, then the problem (1) possesses a nonnegative nontrivial weak solution.

We mention the sublinear case at the end of this paper.

We consider an approximate equation of (2) and seek for a nonnegative weak solution of (2) as a limit of the positive approximate solutions. The LeraySchauder degree theory is adapted to solve the approximate equation. We need an upper bound for positive solutions of equations appearing in certain homotopy to calculate the value of degree in a large ball. An a prori upper estimate for solutions is crucial in this paper. Estimates in $L^{\infty}$ were obtained for global solutions of initial value problems, for example in [5], [14] and [15], but they depend on initial values. We have to derive a priori estimates not depending on initial values. Our tool to get an upper bound is the blow-up (or scaling) argument which was used in [3], [4] and [7]. Our blow-up argument is more complicated since (2) is quasilinear and not semilinear. We make use of inequalities of Harnack type in time and in the space direction for the homotopic equations which are derived in Section 2. Section 3 and Section 4 are devoted to proofs of Theorem 1 and Theorem 2, respectively.

2. Harnack type inequalities. Let $w \in C^{2,1}(\bar{\Omega} \times \mathbb{R})$ be a solution of

$$
\begin{cases}w_{t}=m w^{\gamma}\{\Delta w+g(w, x, t)\} & \text { in } \Omega \times \mathbb{R}, \\ w=\varepsilon & \text { on } \partial \Omega \times \mathbb{R}, \\ w(t+T)=w(t) & \text { in } \Omega \times \mathbb{R},\end{cases}
$$

with $\Delta w_{t} \in C(\bar{\Omega} \times \mathbb{R})$ and $w>\varepsilon$ in $\Omega \times \mathbb{R}$. Similar inequalities of Harnack type were derived in [8] in the case of $\gamma \geq 1$, but they cannot apply to our situation because of $\gamma<1$ in this paper. Put $z=w_{t}$. Differentiating (7) in $t$ yields

$$
z_{t}=\frac{\gamma}{w} z^{2}+m w^{\gamma} \Delta z+m w^{\gamma} g_{w} z+m w^{\gamma} g_{t}
$$

where $g_{w}$ and $g_{t}$ denote the derivatives of $g$ with respect to $w$ and $t$, respectively. Letting $z\left(x_{0}, t_{0}\right)=\min _{\bar{\Omega} \times \mathbb{R}} z$, we have

$$
\gamma z^{2}+m w^{\gamma+1} g_{w} z+m w^{\gamma+1} g_{t} \leq 0 \quad \text { at }\left(x_{0}, t_{0}\right)
$$

and hence

$$
\min z \geq-\frac{m\left(g_{w}\right)_{+}}{\gamma} M^{\gamma+1}-\left(\frac{m}{\gamma}\left\|g_{t}\right\|_{\infty}\right)^{1 / 2} M^{(\gamma+1) / 2},
$$

where $M=\max _{\bar{\Omega} \times \mathbb{R}} w, f_{+}=\max (f, 0)$ and $\|\cdot\|_{\infty}$ denotes the supremum norm. 
Lemma 1. If $w \in C^{2,1}(\bar{\Omega} \times \mathbb{R})$ is a solution of (7) with $\Delta w_{t} \in C(\bar{\Omega} \times \mathbb{R})$ and $w>\varepsilon$ in $\Omega \times \mathbb{R}$, then

$$
w(x, t) \geq w(x, s)-C_{M}(t-s)
$$

for all $s, t \in \mathbb{R}$ with $s \leq t$ and $x \in \bar{\Omega}$, where

$$
\begin{aligned}
C_{M} & =\frac{m}{\gamma} G_{1} M^{\gamma+1}+\left(\frac{m}{\gamma}\right)^{1 / 2} G_{2} M^{(\gamma+1) / 2}, \quad M=\max w ; \\
G_{1} & =\max \left\{\left(g_{w}\right)_{+}: 0<w \leq M,(x, t) \in \bar{\Omega} \times \mathbb{R}\right\}, \\
G_{2} & =\max \left\{\left\|g_{t}\right\|_{\infty}^{1 / 2}: 0<w \leq M,(x, t) \in \bar{\Omega} \times \mathbb{R}\right\} .
\end{aligned}
$$

Proof. Since $w_{t} \geq-C_{M}$ in $\bar{\Omega} \times \mathbb{R}$ from (8), it suffices to integrate this inequality over $(s, t)$.

Lemma 2. Let $N=1$. If $w \in C^{2,1}(\bar{\Omega} \times \mathbb{R})$ is a solution of (7) with $\Delta w_{t} \in C(\bar{\Omega} \times R)$ and $w>\varepsilon$ in $\Omega \times \mathbb{R}$, then

$$
w^{\gamma+1}\left(x, t_{0}\right) \geq w^{\gamma+1}\left(x_{0}, t_{0}\right)-\frac{\gamma+1}{2} \tilde{C}_{M}\left(x-x_{0}\right)^{2} \quad \text { for } x \in \bar{\Omega},
$$

where

$$
\begin{aligned}
\tilde{C}_{M} & =\frac{1}{m} C_{M}+M^{\gamma} G_{3}, \quad M=\max w=w\left(x_{0}, t_{0}\right), \\
G_{3} & =\max \{g: 0<w \leq M,(x, t) \in \bar{\Omega} \times \mathbb{R}\} .
\end{aligned}
$$

Proof. Fix $t \in \mathbb{R}$ arbitrarily. From (8), it follows that

$$
w^{\gamma} w_{x x}=\frac{1}{m} w_{t}-w^{\gamma} g \geq-\tilde{C}_{M}
$$

and hence

$$
\left(w^{\gamma+1}\right)_{x x}=(\gamma+1)\left(\gamma w^{\gamma-1} w_{x}^{2}+w^{\gamma} w_{x x}\right) \geq-(\gamma+1) \tilde{C}_{M} \quad \text { in } \Omega .
$$

Integrating this inequality over $\left(x_{0}, x\right)$ and then the obtained inequality over $\left(x_{0}, x\right)$, we have the desired inequality.

Lemma 3. Let $N \geq 2$. If $w \in C^{2,1}(\bar{\Omega} \times \mathbb{R})$ is a solution of (7) with $\Delta w_{t} \in C(\bar{\Omega} \times \mathbb{R})$ and $w>\varepsilon$ in $\Omega \times \mathbb{R}$, then

$$
w^{\gamma+1}\left(x_{0}, t\right) \leq \frac{1}{\omega_{N} \rho^{N}} \int_{B_{\rho}\left(x_{0}\right)} w^{\gamma+1}(x, t) d x+\frac{(\gamma+1) \tilde{C}_{M}}{2(N+2)} \rho^{2}
$$

for each $x_{0} \in \Omega, t \in \mathbb{R}$ and $0<\rho<\operatorname{dist}\left(x_{0}, \partial \Omega\right)$, where $\omega_{N}$ denotes the volume of unit balls in $\mathbb{R}^{N}$. 
Proof. Fix $t \in \mathbb{R}$ arbitrarily. We get

$$
\Delta w^{\gamma+1} \geq-(\gamma+1) \tilde{C}_{M}
$$

in the same way as in (9). That is, it holds that

$$
\Delta\left(w^{\gamma+1}+\frac{(\gamma+1) \tilde{C}_{M}}{2 N}\left\|x-x_{0}\right\|^{2}\right) \geq 0
$$

for every $x_{0} \in \Omega$. According to the mean value theorem, it follows that for each $0<\rho<\operatorname{dist}\left(x_{0}, \partial \Omega\right)$

$$
\begin{aligned}
\omega^{\gamma+1}\left(x_{0}, t\right) & \leq \frac{1}{\omega_{N} \rho^{N}} \int_{B_{\rho}\left(x_{0}\right)}\left\{\omega^{\gamma+1}(x, t)+\frac{(\gamma+1) \tilde{C}_{M}}{2 N}\left\|x-x_{0}\right\|^{2}\right\} d x \\
& =\frac{1}{\omega_{N} \rho^{N}} \int_{B_{\rho}\left(x_{0}\right)} \omega^{\gamma+1}(x, t) d x+\frac{(\gamma+1) \tilde{C}_{M}}{2(N+2)} \rho^{2} .
\end{aligned}
$$

3. Proof of Theorem 1. To solve the problem (2), we consider the following approximate equation

$$
\begin{cases}v_{t}=m(v+\varepsilon)^{\gamma}\{\Delta v+h(t) \tilde{f}(u)\} & \text { in } \Omega \times \mathbb{R} \\ v=0 & \text { on } \partial \Omega \times \mathbb{R} \\ v(t+T)=v(t) & \text { in } \Omega \times \mathbb{R}\end{cases}
$$

with $\varepsilon>0$. Fix a positive constant $k$. Set

$$
\Phi(\rho, x, t)=k \rho_{+}+h(t) \tilde{f}\left(\rho_{+}\right)
$$

and

$$
\tilde{\Phi}(\rho, x, t)=k \rho_{+}+\lambda_{1} \rho_{+}+h(t) \tilde{f}\left(\rho_{+}\right)+1
$$

for all $(\rho, x, t) \in \mathbb{R} \times \bar{\Omega} \times \mathbb{R}$. The Leray-Schauder degree theory is adapted to obtain approximate solutions. As seen later, to calculate the value of degree in a large ball, we need an a priori upper bound for positive solutions of

$$
\begin{cases}v_{t}=m(v+\varepsilon)^{\gamma}\{\Delta v-k v+\tau \Phi(v)+(1-\tau) \tilde{\Phi}(v)\} & \text { in } \Omega \times \mathbb{R}, \\ v=0 & \text { on } \partial \Omega \times \mathbb{R}, \\ v(t+T)=v(t) & \text { in } \Omega \times \mathbb{R},\end{cases}
$$

with $\tau \in[0,1]$. By standard regularity theory and the maximum principle, every solution $v$ is a classical positive solution and satisfies $\Delta v_{t} \in C(\bar{\Omega} \times \mathbb{R})$ under the 
regularity assumptions of $f$ and $h$. Putting $w=v+\varepsilon$ for each positive solution $v$ of (12), $w$ satisfies

$$
\begin{cases}w_{t}=m w^{\gamma}\left\{\Delta w+h(t) \tilde{f}(w-\varepsilon)+(1-\tau) \lambda_{1} w\right. & \text { in } \Omega \times \mathbb{R}, \\ w=\varepsilon & \text { on } \partial \Omega \times \mathbb{R}, \\ w(t+T)=w(t) & \text { in } \Omega \times \mathbb{R},\end{cases}
$$

with $\tau \in[0,1]$. From the assumption (3), $\tilde{f}$ is written as

$$
\tilde{f}(\rho)=c \rho^{q}+\varphi(\rho) \quad \text { for } \rho \geq 0
$$

with $c>0, q=p / m$ and $\varphi \in o\left(\rho^{q}\right)$ as $\rho \rightarrow \infty$. By the hypotheses on $h$, there are $\alpha, \beta>0$ such that

$$
\alpha \leq h(t) \leq \beta \quad \text { for all } t \in \mathbb{R} .
$$

Let $Q=\Omega \times(0, T)$.

Lemma 4. Under the assumptions of Theorem 1, there exist positive constants $K_{1}, K_{2}, K_{3}$, such that

$$
\iint_{Q} w(x, t)^{q} d x d t \leq K_{1} \quad \text { and } \quad \iint_{Q} \frac{w_{t}^{2}}{w^{\gamma}} d x d t \leq K_{2} M+K_{3}
$$

for each solution $w \in C^{2,1}(\bar{\Omega} \times \mathbb{R})$ of (13) with $w>\varepsilon$ in $\Omega \times \mathbb{R}$ and $0<\varepsilon \leq 1$, where $M=\max w$.

Proof. It suffices to prove the existence of $C_{1}, C_{2}, C_{3}>0$ such that

$$
\iint_{Q} v^{q} d x d t \leq C_{1} \quad \text { and } \quad \iint_{Q} \frac{v_{t}^{2}}{(v+\varepsilon)^{\gamma}} d x d t \leq C_{2} M+C_{3}
$$

for each positive solution $v$ of (12) and $0<\varepsilon \leq 1$. Let $\phi_{1}$ be a positive eigenfunction corresponding to $\lambda_{1}$. Multiplying $\phi_{1} /(v+\varepsilon)^{\gamma}$ with (12) and integrating over $Q$ yields

$$
\iint_{Q}\left\{-\lambda_{1} v+h(t) \tilde{f}(v)+(1-\tau)\left(\lambda_{1} v+1\right)\right\} \phi d x d t=0 .
$$

Since $|\varphi(\rho)| \leq(\alpha c / 2 \beta) \rho^{q}+c_{0}$ for all $\rho \geq 0$ with some $c_{0}>0$ from $\varphi \in o\left(\rho^{q}\right)$ as $\rho \rightarrow \infty$, we get

$$
\alpha c \iint_{Q} v^{q} \phi_{1} d x d t \leq \iint_{Q}\left\{\lambda_{1} v+\beta\left(\frac{\alpha c}{2 \beta} v^{q}+c_{0}\right)\right\} \phi_{1} d x d t
$$


so there is $c_{1}>0$ such that

$$
\iint_{Q} v^{q} \phi_{1} d x d t \leq c_{1}
$$

By the same argument as Step 2 in the proof of Theorem 1.1 in [2], there are positive constants $\delta_{0}, c_{2}$ such that

$$
v \leq c_{2} \quad \Omega_{\delta_{0}} \times \mathbb{R}
$$

for each positive solution $v$ of (12), where $\Omega_{\delta_{0}}=\left\{x \in \Omega\right.$ : $\left.\operatorname{dist}(x, \partial \Omega)<\delta_{0}\right\}$. Therefore, we obtain some positive constant $C_{1}$ such that

$$
\iint_{Q} v^{q} d x d t \leq C_{1}
$$

for each positive solution $v$ of (12). On the other hand, multiplying $v_{t} /(v+\varepsilon)^{\gamma}$ with (12) and integrating over $Q$ yields

$$
\iint_{Q} \frac{v_{t}^{2}}{(v+\varepsilon)^{\gamma}} d x d t=-m \iint h_{t} \tilde{F}(v) d x d t
$$

where

$$
\tilde{F}(\rho)=\int_{0}^{\rho} \tilde{f}(r) d r \quad \text { for } \rho \geq 0 .
$$

Since the growth condition of $\tilde{f}$ implies the existence of some $c_{3}, c_{4}>0$ such that

$$
\tilde{F}(\rho) \leq c_{3} \rho^{q+1}+c_{4} \text { for all } \rho \geq 0,
$$

we derive the second inequality.

Lemma 5. Let $N=1$. Under the hypotheses of Theorem 1, there exist positive constants $\delta_{1}, \rho_{1}, C_{1}$ such that if $w \in C^{2,1}(\bar{\Omega} \times \mathbb{R})$ is a solution of (13) with $w_{x x t} \in C(\bar{\Omega} \times \mathbb{R}), w>\varepsilon$ in $\Omega \times \mathbb{R}$ and $M=\max w=w\left(x_{0}, t_{0}\right)>C_{1}$, then

$$
\begin{aligned}
& w(x, t) \geq \frac{1}{4} M \quad \text { for } x \in \mathbb{R} \text { with }\left|x-x_{0}\right| \leq \delta_{1} M^{(1-q) / 2} \text { and } \\
& \qquad t_{0} \leq t \leq t_{0}+\rho_{1} M^{1-\gamma-q} .
\end{aligned}
$$

Proof. Set

$$
g(\rho, x, t)=h(t) \tilde{f}(\rho-\varepsilon)+(1-\tau) \lambda_{1} \rho+(1-\tau)\left(1-\lambda_{1} \varepsilon\right) .
$$


By the assumptions (3), (4), we have positive constants $c_{0}, c_{1}, c_{2}$ such that

$$
C_{M} \leq c_{0} M^{\gamma+q} \quad \text { and } \quad \tilde{C}_{M} \leq c_{1} M^{\gamma+q}
$$

if $M \geq c_{2}$, where $C_{M}$ and $\tilde{C}_{M}$ are as in Lemma 2 . Set

$$
\delta_{1}=\left\{\frac{2}{c_{1}(\gamma+1)}\left(1-\left(\frac{1}{2}\right)^{\gamma+1}\right)\right\}^{1 / 2} .
$$

Since $x_{0}$ is the middle point of $\Omega$ from [6], $\left\{x \in \mathbb{R}:\left|x-x_{0}\right| \leq \delta_{1} M^{(1-q) / 2}\right\} \subset$ $\Omega$ if $M>\left(2 \delta_{1} /|\Omega|\right)^{2 /(q-1)}$, where $|\Omega|$ denotes the length of $\Omega$. Put $C_{1}=$ $\max \left(c_{2},\left(2 \delta_{1} /|\Omega|\right)^{2 /(q-1)}\right)$. From Lemma 2 , for each $x \in \mathbb{R}$ with $\left|x-x_{0}\right| \leq$ $\delta_{1} M^{(1-q) / 2}$, we have

$$
w^{\gamma+1}\left(x, t_{0}\right) \geq M^{\gamma+1}-\frac{\gamma+1}{2} c_{1} M^{\gamma+q}\left(x-x_{0}\right)^{2} \geq\left(\frac{M}{2}\right)^{\gamma+1},
$$

that is,

$$
w\left(x, t_{0}\right) \geq \frac{M}{2} \quad \text { for each } x \in \mathbb{R} \text { with }\left|x-x_{0}\right| \leq \delta_{1} M^{(1-q) / 2} .
$$

Putting $\rho_{1}=1 / 4 c_{0}$, Lemma 1 implies

$$
w(x, t) \geq w\left(x, t_{0}\right)-c_{0} M^{\gamma+q}\left(t-t_{0}\right) \geq \frac{1}{4} M
$$

if $M>C_{1},\left|x-x_{0}\right| \leq \delta_{1} M^{(1-q) / 2}$ and $t_{0} \leq t \leq t_{0}+\rho_{1} M^{1-\gamma-q}$.

Lemma 6. Let $N \geq 2$. Under the hypotheses of Theorem 1, there exist positive constants $\delta_{2}, \rho_{2}, C_{2}$ such that if $w \in C^{2,1}(\bar{\Omega} \times \mathbb{R})$ is a solution of (13) with $\Delta w_{t} \in C(\bar{\Omega} \times \mathbb{R}), w>\varepsilon$ in $\Omega \times \mathbb{R}$ and $M=\max w=w\left(x_{0}, t_{0}\right)>C_{2}$,

$$
\int_{B_{\delta}\left(x_{0}\right)} w^{\gamma+1}(x, t) d x \geq\left(\frac{1}{2}\right)^{\gamma+2} \omega_{N} \delta^{N} M^{\gamma+1}
$$

for $0<\delta \leq \delta_{2} M^{(1-q) / 2}$ and $t_{0} \leq t \leq t_{0}+\rho_{2} M^{1-\gamma-q}$.

Proof. Let $c_{0}, c_{1}, c_{2}$ be as in the proof of Lemma 5. Putting $\rho_{2}=1 / 2 c_{0}$, Lemma 1 implies

$$
w\left(x_{0}, t\right) \geq \frac{M}{2} \quad \text { for } t_{0} \leq t \leq t_{0}+\rho_{2} M^{1-\gamma-q} .
$$

Set $\delta_{2}=\left\{\left((N+2) /(\gamma+1) c_{1}\right)\left(\frac{1}{2}\right)^{\gamma+1}\right\}^{1 / 2}$. Since $\Omega$ is convex, there is $\delta_{0}>0$ such that $\operatorname{dist}\left(x_{0}, \partial \Omega\right) \geq \delta_{0}$ by [6], so $B_{\delta}\left(x_{0}\right) \subset \Omega$ if $\delta \leq \delta_{2} M^{(1-q) / 2}$ and $M>$ 
$\left(\delta_{2} / \delta_{0}\right)^{2 /(q-1)}$. Putting $C_{2}=\max \left(c_{2},\left(\delta_{2} / \delta_{0}\right)^{2 /(q-1)}\right)$, Lemma 3 yields the desired inequality if $M>C_{2}, 0<\delta \leq \delta_{2} M^{(1-q) / 2}$ and $t_{0} \leq t \leq t_{0}+\rho_{2} M^{1-\gamma-q}$.

We now derive upper bounds for solutions of (13) by the blow-up argument. The properties of solutions of (13) obtained in [6] play an important part in Lemmas 7-9.

Lemma 7. Let $\Omega$ be an open interval in $\mathbb{R}$. Under the assumptions of Theorem 1 , there is $M_{0}>0$ such that $\max w \leq M_{0}$ for every solution $w \in$ $C^{2,1}(\bar{\Omega} \times \mathbb{R})$ of $(13)$ with $w_{x x t} \in C(\bar{\Omega} \times \mathbb{R})$ and $w>\varepsilon$ in $\Omega \times \mathbb{R}$ and $0<\varepsilon \leq 1$.

Proof. Fix $0<\varepsilon \leq 1$. Assuming that there are no upper bounds, we get a sequence $\left\{w_{n}\right\} \subset C^{2,1}(\bar{\Omega} \times \mathbb{R})$ of solutions of $(13)$ with $\Delta w_{n t} \in C(\bar{\Omega} \times \mathbb{R})$ and $w_{n}>\varepsilon$ in $\Omega \times \mathbb{R}$ such that $M_{n}=\max w_{n}=w_{n}\left(x_{n}, t_{n}\right) \rightarrow \infty$ as $n \rightarrow \infty$. By [6], $x_{n}$ is the middle point of $\Omega$ and $w_{n}(\cdot, t)$ is symmetric with respect to $x=x_{n}$ and strictly decreasing in $x>x_{n}$ for each $t \in \mathbb{R}$ and $n \in \mathbb{N}$. Therefore, we may assume that $\Omega=(-a, a)$ without loss of generality. Let $k=(q-1) / 2$ and $\ell=q+\gamma-1$. Put $\lambda_{n}=1 / M_{n}, B_{n}=\left\{y \in \mathbb{R}:|y|<a / \lambda_{n}^{k}\right\}$ and $Q_{n}=B_{n} \times \mathbb{R}$. Define $v_{n}: Q_{n} \rightarrow \mathbb{R}$ by

$$
v_{n}(y, s)=\lambda_{n} w_{n}\left(\lambda_{n}^{k} y, t_{n}+\lambda_{n}^{\ell} s\right) \quad \text { for }(y, s) \in Q_{n} .
$$

Then it is immediate that $0<v_{n} \leq 1$ and $\max v_{n}=1=v_{n}(0,0)$. By straightforward calculation, we derive

$$
v_{n s}=m v_{n}^{\gamma}\left\{v_{n y y}+\operatorname{ch}\left(t_{n}+\lambda_{n}^{\ell} s\right) v_{n}^{q}+\lambda_{n}^{q} \tilde{\varphi}\left(\lambda_{n}^{-1} v_{n}\right)\right\},
$$

where $\tilde{\varphi} \in o\left(\rho^{q}\right)$ as $\rho \rightarrow \infty$, i.e., for any $\varepsilon>0,|\tilde{\varphi}(\rho)| \leq \varepsilon \rho^{q}+C_{\varepsilon}$ for all $\rho \geq 0$ with some $C_{\varepsilon}>0$. Therefore, it holds that

$$
\begin{aligned}
& -\frac{1}{1-\gamma} \iint v_{n}^{1-\gamma} \psi_{s} d y d s \\
= & m \iint\left\{v_{n} \psi_{y y}+\operatorname{ch}\left(t_{n}+\lambda_{n}^{\ell} s\right) v_{n}^{q} \psi+\lambda_{n}^{q} \tilde{\varphi}\left(\lambda_{n}^{-1} v_{n}\right) \psi\right\} d y d s
\end{aligned}
$$

for each $\psi \in C_{0}^{\infty}\left(\mathbb{R}^{2}\right)$. Fix $R>0$ arbitrarily. By Lemma 4 , we get

$$
\begin{aligned}
\int_{-R}^{R} \int_{-R}^{R}\left(v_{n}^{1+\gamma}\right)_{s}^{2} d y d s & \leq(1+\gamma)^{2} \int_{-R}^{R} \int_{-R}^{R} v_{n s}^{2} d y d s \\
& =(1+\gamma)^{2} \lambda_{n}^{\ell+2-k} \int_{t_{n}-\lambda_{n}^{\ell} R}^{t_{n}+\lambda_{n}^{\ell} R} \int_{-\lambda_{n}^{k} R}^{\lambda_{n}^{k} R} w_{n t}^{2} d x d t \\
& \leq 2 K_{2} \lambda_{n}^{\ell+1-k-\gamma}+2 K_{3} \lambda_{n}^{\ell+2-k-\gamma} .
\end{aligned}
$$


From $\ell+1-k-\gamma>0$, it follows that

$$
\int_{-R}^{R} \int_{-R}^{R}\left(v_{n}^{1+\gamma}\right)_{s}^{2} d y d s \rightarrow 0 \quad \text { as } n \rightarrow \infty .
$$

On the other hand, for $0<x \leq \lambda_{n}^{k} R$, integrating (9) over $(0, x)$ yields

$$
\left(w_{n}^{\gamma+1}\right)_{x}(x, t) \geq-(\gamma+1) c_{1} M_{n}^{\gamma+q} x \geq-(\gamma+1) c_{1} \lambda_{n}^{k-\gamma-q} R,
$$

since $\tilde{C}_{M_{n}} \leq c_{1} M_{n}^{\gamma+q}$ for all $n$ with some $c_{1}>0$. From $\left(w_{n}^{\gamma+1}\right)_{x}(x, t)<0$, for $x>0$ and the symmetry of $w_{n}(\cdot, t)$ with respect to $x=0$, we get

$$
\int_{-\lambda_{n}^{k} R}^{\lambda_{n}^{k} R} \int_{t_{n}-\lambda_{n}^{\ell} R}^{t_{n}+\lambda_{n}^{\ell} R}\left(w_{n}^{\gamma+1}\right)_{x}^{2} d x d t \leq 4 R^{2}(\gamma+1)^{2} c_{1}^{2} \lambda_{n}^{3 k-2 \gamma-2 q+\ell} \quad \text { for all } n .
$$

Therefore, we have

$$
\int_{-R}^{R} \int_{-R}^{R}\left(v_{n}^{\gamma+1}\right)_{y}^{2} d y d s \leq 4(\gamma+1)^{2} R^{2} c_{1}^{2}
$$

By this boundedness of $\left\{v_{n}^{\gamma+1}\right\}$ in $H_{\mathrm{loc}}^{1}\left(\mathbb{R}^{2}\right)$, we may assume that $\left\{v_{n}^{\gamma+1}\right\}$ converges to some nonnegative function $\xi$ weakly in $H_{\text {loc }}^{1}\left(\mathbb{R}^{2}\right)$ and a.e. in $\mathbb{R}^{2}$. Putting $v=\xi^{1 /(\gamma+1)}, v_{n} \rightarrow v$ a.e. in $\mathbb{R}^{2}$. Letting $n \rightarrow \infty$ in (18) yields

$$
-\frac{1}{1-\gamma} \iint v^{1-\gamma} \psi_{s} d y d s=m \iint\left\{v \psi_{y y}+\operatorname{ch}\left(t_{0}\right) v^{q} \psi\right\} d y d s
$$

for all $\psi \in C_{0}^{\infty}\left(\mathbb{R}^{2}\right)$, where $t_{0}=\lim _{n \rightarrow \infty} t_{n}$. From $\left(v^{\gamma+1}\right)_{s}=0$ a.e. in $\mathbb{R}^{2}, v^{\gamma+1}$ is independent of $s$ and hence so is $v$. Therefore, it holds that

$$
\iint\left\{v \tilde{\psi}_{y y}+\operatorname{ch}\left(t_{0}\right) v^{q} \tilde{\psi}\right\} d y d s=0
$$

for all $\tilde{\psi} \in C_{0}^{\infty}(\mathbb{R})$. According to the standard regularity theory, $v$ satisfies

$$
v_{y y}+\operatorname{ch}\left(t_{0}\right) v^{q}=0 \quad \text { in } \mathbb{R}
$$

in the classical sense. By Liouville's theorem, this implies $v \equiv 0$. On the other hand, Lemma 5 yields

$$
\text { for } y \text { with }|y| \leq \delta_{1} \operatorname{and}(9, \S) \unrhd_{4}^{1} \rho_{1} \text {. }
$$

This is a contradiction. 
Lemma 8. Let $\Omega$ be an open ball in $\mathbb{R}^{2}$. Under the assumptions of Theorem 1 , there is $M_{0}>0$ such that $\max w \leq M_{0}$ for each solution $w \in C^{2,1}(\bar{\Omega} \times \mathbb{R})$ of (13) with $\Delta w_{t} \in C(\bar{\Omega} \times \mathbb{R})$ and $w>\varepsilon$ in $\Omega \times \mathbb{R}$ and $0<\varepsilon \leq 1$.

Proof. Without loss of generality, we may assume that $\Omega=B_{a}(0)$. Assume that there are no upper bounds and let $\left\{w_{n}\right\}$ be as in the proof of Lemma 7. By [6], for $n \in \mathbb{N}$, and $t \in \mathbb{R}, w_{n}(\cdot, t)$ is radially symmetric and $w_{n}(\rho, t)$ is strictly decreasing in $\rho$, where $\rho=\|x\|$. From (10),

$$
\Delta w_{n}^{\gamma+1} \geq-(\gamma+1) c_{1} M_{n}^{\gamma+q} \text { in } \Omega
$$

with some $c_{1}>0$. Since

$$
\Delta w_{n}^{\gamma+1}=\left(w_{n}^{\gamma+1}\right)_{\rho \rho}+\frac{1}{\rho}\left(w_{n}^{\gamma+1}\right)_{\rho}
$$

we get

$$
\left(\rho\left(w_{n}^{\gamma+1}\right)_{\rho}\right)_{\rho} \geq-(\gamma+1) c_{1} M_{n}^{\gamma+q} \rho \quad \text { for } 0<\rho<a
$$

and hence,

$$
\left(w_{n}^{\gamma+1}\right)_{\rho}(\rho) \geq-\frac{(\gamma+1) c_{1}}{2} M_{n}^{\gamma+q} \rho \quad \text { for } 0<\rho<a .
$$

It follows that

$$
\left|\nabla w_{n}^{\gamma+1}\right|^{2}=\left(w_{n}^{\gamma+1}\right)_{\rho}^{2} \leq \frac{(\gamma+1)^{2} c_{1}^{2}}{4} M_{n}^{2(\gamma+q)} \rho^{2}
$$

for $0<\rho<a$ since $w_{n \rho}(\rho, t)<0$ for $0<\rho<a$ and $t \in \mathbb{R}$. Put

$$
\lambda_{n}=\frac{1}{M_{n}}, \quad B_{n}=\left\{y \in \mathbb{R}^{2}:\|y\| \leq \frac{a}{\lambda_{n}^{k}}\right\} \quad \text { and } \quad Q_{n}=B_{n} \times \mathbb{R} .
$$

Defining $v_{n}: Q_{n} \rightarrow \mathbb{R}$ by (16), we can show this lemma in the same way as in the proof of Lemma 7 .

Lemma 9. Let $N \geq 2$ and $m<p<(N m+2) / N$. Under the assumptions of Theorem 1, there is $M_{0}>0$ such that $\max w \leq M_{0}$ for every solution $w \in$ $C^{2,1}(\bar{\Omega} \times \mathbb{R})$ of (13) with $\Delta w_{t} \in C(\bar{\Omega} \times \mathbb{R})$ and $w>\varepsilon$ in $\Omega \times \mathbb{R}$ and $0<\varepsilon \leq 1$. 
Proof. Assuming that there are no upper bounds, let $\left\{w_{n}\right\}$ and $\left\{\left(x_{n}, t_{n}\right)\right\}$ be as in the proof of Lemma 7. By the convexity of $\Omega$, we obtain $\delta_{0}>0$ such that

$$
\operatorname{dist}\left(x_{n}, \partial \Omega\right) \geq \delta_{0} \quad \text { for all } n
$$

from [6]. Put $\lambda_{n}=1 / M_{n}, B_{n}=\left\{y \in \mathbb{R}^{N}:\left\|y-x_{n}\right\| \leq \delta_{0} / \lambda_{n}^{k}\right\}$ and $Q_{n}=B_{n} \times \mathbb{R}$. Defining $v_{n}: Q_{n} \rightarrow \mathbb{R}$ by

$$
v_{n}(y, s)=\lambda_{n} w_{n}\left(x_{n}+\lambda_{n}^{k} y, t+\lambda_{n}^{\ell} s\right) \quad \text { for }(y, s) \in Q_{n},
$$

it holds that

$$
\left(\frac{1}{1-\gamma} v_{n}^{1-\gamma}\right)_{s} \geq m\left\{\Delta v_{n}+(c a-\varepsilon) v_{n}^{q}-C_{\varepsilon} \lambda_{n}^{q}\right\}
$$

by the same argument as in the proof of Lemma 7 . Taking $\varepsilon=c \alpha / 2$, we get

$$
\left(\frac{1}{1-\gamma} v_{n}^{1-\gamma}\right)_{s} \geq m\left\{\Delta v_{n}+\frac{c \alpha}{2} v_{n}^{q}-C_{\varepsilon} \lambda_{n}^{q}\right\}
$$

For each $R>0$, let $\nu_{R}$ and $\phi_{R}$ denote the first eigenvalue of $-\Delta$ in $B_{R}(0) \subset \mathbb{R}^{N}$ with the Dirichlet boundary condition and the eigenfunction corresponding to $\nu_{R}$ with $\int_{B_{R}(0)} \phi_{R} d y=1$. Then it is easily seen that

$$
\nu_{R}=\frac{\nu_{1}}{R^{2}} \quad \text { and } \quad \phi_{R}(y)=\frac{1}{R^{N}} \phi_{1}\left(\frac{y}{R}\right) .
$$

Multiplying $\phi_{R}$ with (19) and integrating over $B_{R}(0)$ yields

$$
\frac{d}{d s} \int_{B_{R}(0)} \frac{1}{1-\gamma} v_{n}^{1-\gamma} \phi_{R} d y \geq m \int_{B_{R}(0)}\left\{-\nu_{R} v_{n} \phi_{R}+\frac{c \alpha}{2} v_{n}^{q} \phi_{R}-C_{\varepsilon} \lambda_{n}^{q} \phi_{R}\right\} d y .
$$

By Hölder's inequality, we have

$$
\begin{aligned}
& \frac{1}{1-\gamma} \frac{d}{d s} \int_{B_{R}(0)} v_{n}^{1-\gamma} \phi_{R} d y \\
& \geq m \int_{B_{R}(0)} v_{n} \phi_{R} d y\left\{-\nu_{R}+\frac{c \alpha}{2}\left(\int_{B_{R}(0)} v_{n}^{1-\gamma} \phi_{R} d y\right)^{(q-1) /(1-\gamma)}\right. \\
& \\
&\left.\quad-C_{\varepsilon} \lambda_{n}^{q}\left(\int_{B_{r}(0)} v_{n}^{1-\gamma} \phi_{R} d y\right)^{-1 /(1-\gamma)}\right\} .
\end{aligned}
$$


Setting

$$
\xi(s)=\int_{B_{R}(0)} v_{n}^{1-\gamma} \phi_{R} d y
$$

for $s \in \mathbb{R}$, it holds that

$$
\frac{1}{1-\gamma} \frac{d \xi}{d s} \geq m \int_{B_{r}(0)} v_{n} \phi_{R} d y\left(-\nu_{R}+\frac{c \alpha}{2} \xi^{(q-1) /(1-\gamma)}-C_{\varepsilon} \lambda_{n}^{q} \xi^{-1 /(1-\gamma)}\right) .
$$

Suppose that

$$
-\nu_{R}+\frac{c \alpha}{2} \xi^{(q-1) /(1-\gamma)}-C_{\varepsilon} \lambda_{n}^{q} \xi^{-1 /(1-\gamma)}>0
$$

at some $s_{0} \in \mathbb{R}$. Then we get

$$
\frac{1}{1-\gamma} \frac{d \xi}{d s} \geq m \xi^{1 /(1-\gamma)}\left(-\nu_{R}+\frac{c \alpha}{2} \xi^{(q-1) /(1-\gamma)}-C_{\varepsilon} \lambda_{n}^{q} \xi^{-1 /(1-\gamma)}\right)
$$

for $s \geq s_{0}$. This implies that $\xi$ cannot be bounded on $\mathbb{R}$. Therefore, it follows from the boundedness of $\xi$ on $\mathbb{R}$ that

$$
-\nu_{R}+\frac{c \alpha}{2} \xi^{(q-1) /(1-\gamma)}-C_{\varepsilon} \lambda_{n}^{q} \xi^{-1 /(1-\gamma)} \leq 0 \quad \text { for all } s \in \mathbb{R},
$$

that is,

$$
\begin{aligned}
-\nu_{R}+ & \frac{c \alpha}{2}\left(\int_{B_{R}(0)} v_{n}^{1-\gamma} \phi_{R} d y\right)^{(q-1) /(1-\gamma)} \\
& -C_{\varepsilon} \lambda_{n}^{q}\left(\int_{B_{R}(0)} v_{n}^{1-\gamma} \phi_{R} d y\right)^{-1 /(1-\gamma)} \leq 0
\end{aligned}
$$

for all $s \in \mathbb{R}$. Letting $\rho_{2}, \delta_{2}$ be as in Lemma 6 , we see

$$
\int_{B_{\delta_{2}}(0)} v_{n}^{1-\gamma} d y \geq\left(\frac{1}{2}\right)^{\gamma+2} \omega_{N} \delta_{2}^{N}
$$

for $0<s<\rho_{2}$. We also get

$$
\int_{B_{R}(0)} v_{n}^{1-\gamma} \phi_{R} d y \geq c_{1} R^{-N} \int_{B_{R / 2}(0)} v_{n}^{1-\gamma} d y
$$

where $c_{1}$ is the value of $\phi_{1}$ on $\partial B_{1 / 2}(0)$. It follows that

$$
\int_{B_{R}(0)} v_{n}^{1-\gamma} \phi_{R} d y \geq\left(\frac{1}{2}\right)^{\gamma+2} c_{1} \omega_{N} \delta_{2}^{N} R^{-N}
$$


for $R \geq 2 \delta_{2}$. Therefore, we derive

$$
-\nu_{R}+\frac{c \alpha}{2}\left(\int_{B_{R}(0)} v_{n}^{1-\gamma} \phi_{R} d y\right)^{(q-1) /(1-\gamma)}-C_{\varepsilon} \lambda_{n}^{q}\left(\frac{2^{\gamma+2} R^{N}}{c_{1} \omega_{N} \delta_{2}^{N}}\right)^{1 /(1-\gamma)} \leq 0 .
$$

Integrating this inequality over $\left(0, \rho_{2}\right)$ and using Hölder's inequality yields

$$
\begin{array}{r}
-\nu_{R}+\frac{c \alpha}{2} \rho_{2}^{(2-q-\gamma) /(1-\gamma)}\left(\int_{0}^{\rho_{2}} \int_{B_{R}(0)} v_{n}^{1-\gamma} \phi_{R} d y d s\right)^{(q-1) /(1-\gamma)} \\
-C_{\varepsilon} \lambda_{n}^{q}\left(\frac{2^{\gamma+2} R^{N}}{c_{1} \omega_{N} \delta_{2}^{N}}\right)^{1 /(1-\gamma)} \leq 0 .
\end{array}
$$

Since $\left\{v_{n}^{1-\gamma}\right\}$ converges to some $w$ in $L^{\infty}$ in the weak* topology, we get

$$
\int_{0}^{\rho_{2}} \int_{B_{R}(0)} w \phi_{R} d y d s \leq c_{2} R^{-2(1-\gamma) /(q-1)}
$$

with some $c_{2}>0$, letting $n \rightarrow \infty$ in (22). In the same way as (21), it holds that

$$
\int_{0}^{\rho_{2}} \int_{B_{R}(0)} w \phi_{R} d y d s \geq c_{1} R^{-N} \int_{0}^{\rho_{2}} \int_{B_{R / 2}(0)} w d y d s
$$

and hence

$$
\int_{0}^{\rho_{2}} \int_{B_{R / 2}(0)} w d y d s \leq \frac{c_{2}}{c_{1}} R^{N-2(1-\gamma) /(q-1)} .
$$

From $p<(N m+2) / N$, it follows that

$$
\int_{0}^{\rho_{2}} \int_{B_{R / 2}(0)} w d y d s \rightarrow 0 \quad \text { as } R \rightarrow \infty
$$

Integrating $(20)$ over $\left(0, \rho_{2}\right)$ yields

$$
\int_{0}^{\rho_{2}} \int_{B_{R / 2}(0)} v_{n}^{1-\gamma} d y d s \geq\left(\frac{1}{2}\right)^{\gamma+2} \omega_{N} \rho_{2} \delta_{2}^{N}
$$

for $R \geq 2 \delta_{2}$, which is a contradiction.

Let $X$ be the space of functions in $C(\bar{\Omega} \times \mathbb{R})$ which are $T$-periodic in time. The following result was given in Lemma 5 in [9]. 
Lemma 10. Assume that $k$ is a positive constant and that a continuous function $a: \mathbb{R} \rightarrow \mathbb{R}$ satisfies

$$
\lambda \leq a(r) \leq \Lambda
$$

for $r \in \mathbb{R}$

with some positive constants $\lambda, \Lambda$. Then for each $u \in X$, there exists a unique solution $v \in \bigcap_{p>1} W_{p}^{2,1}(Q)$ of

$$
\begin{cases}v_{t}=a(v)(\Delta v-k v+u) & \text { in } \Omega \times \mathbb{R}, \\ v=0 & \text { on } \partial \Omega \times \mathbb{R}, \\ v(t+T)=v(t) & \text { in } \Omega \times \mathbb{R} .\end{cases}
$$

Moreover, for any $p>1$, there is $C_{p}>0$ depending only on $\lambda, \Lambda, p$ such that

$$
\|v\|_{W_{p}^{2,1}(Q)} \leq C_{p}\|u\|_{\infty}
$$

For $u \in X$, define $S(u)$ by the unique solution of

$$
\begin{cases}v_{t}=m a_{\varepsilon}(v)(\Delta v-k v+u) & \text { in } \Omega \times \mathbb{R} \\ v=0 & \text { on } \partial \Omega \times \mathbb{R}, \\ v(t+T)=v(t) & \text { in } \Omega \times \mathbb{R}\end{cases}
$$

where $a_{\varepsilon}: \mathbb{R} \rightarrow \mathbb{R}$ is a $C^{1}$-function such that

$$
a_{\varepsilon}(r) \geq \lambda_{\varepsilon} \quad \text { and } \quad 0 \leq \frac{d a_{\varepsilon}}{d r} \leq \gamma \varepsilon^{\gamma-1} \quad \text { for } r \in \mathbb{R}
$$

with some $\lambda_{\varepsilon}>0$, and

$$
a_{\varepsilon}(r)=(r+\varepsilon)^{\gamma} \quad \text { for } r \geq 0 .
$$

Since $\max S(u) \leq\|u\|_{\infty}$ by the maximum principle, $S$ is well-defined and a compact operator from $X$ into itself from Lemma 10 .

Lemma 11. Under the assumptions of Theorem 1, there is $r>0$ such that

$$
\operatorname{deg}\left(I-S \circ \Phi, B_{r}(0), 0\right)=1 \quad \text { for all } \varepsilon>0,
$$

where deg denotes the Leray-Schauder degree and $B_{r}(0)$ is the closed ball with radius $r$ centered at 0 in $X$. 
Proof. We consider

$$
\begin{cases}v_{t}=m a_{\varepsilon}(v)(\Delta v-k v+\tau \Phi(v)) & \text { in } \Omega \times \mathbb{R} \\ v=0 & \text { on } \partial \Omega \times \mathbb{R} \\ v(t+T)=v(t) & \text { in } \Omega \times \mathbb{R}\end{cases}
$$

with $\tau \in[0,1]$. By standard regularity theory and the maximum principle, each nontrivial solution $v$ of $(23)$ is a positive classical solution, so $v$ satisfies

$$
v_{t}=m(v+\varepsilon)^{\gamma}\{\Delta v-(1-\tau) k v+\tau h(t) \tilde{f}(v)\} \quad \text { in } \Omega \times \mathbb{R} .
$$

From the assumption (5), there are positive constants $c_{1}, \rho_{1}$ with $C_{1}<\lambda_{1}$ such that

$$
h(t) \tilde{f}(\rho) \leq c_{1} \rho \text { for all } 0<\rho \leq \rho_{1} \text { and } t \in \mathbb{R} .
$$

Then there is $\delta>0$ such that $\max v \geq \delta$ for each nontrivial solution $v$ of (23). In fact, on the contrary, assume that $\max v_{n}$ converges to 0 as $n \rightarrow \infty$ for some sequence $\left\{v_{n}\right\}$ of nontrivial solutions of (23). Multiplying $\phi_{1} / m\left(v_{n}+\varepsilon\right)^{\gamma}$ with (24) with $v=v_{n}$ and integrating over $Q$ yields

$$
0 \leq \iint_{Q}\left\{\tau c_{1}-\lambda_{1}-(1-\tau) c\right\} v_{n} \phi_{1} d x d t<0
$$

for sufficiently large $n$. This is a contradiction. Taking $r<\delta, S \circ(\tau \Phi)$ has no fixed points on $\partial B_{r}(0)$ for all $\tau \in[0,1]$. According to the homotopy invariance of degree, we obtain

$$
\operatorname{deg}\left(I-S \circ \Phi, B_{r}(0), 0\right)=\operatorname{deg}\left(I, B_{r}(0), 0\right)=1 .
$$

Lemma 12. Under the hypothesis of Theorem 1, there is $R>r$ such that

$$
\operatorname{deg}\left(I-S \circ \Phi, B_{R}(0), 0\right)=0 \quad \text { for all } 0<\varepsilon \leq 1 .
$$

Proof. By Lemma 7-9, there is $M_{0}>0$ such that $\max v \leq M_{0}$ for each positive solution $v$ of $(12)$ and $0<\varepsilon \leq 1$. Taking $R>\max \left(M_{0}, r\right)$, $S \circ(\tau \Phi+(1-\tau) \tilde{\Phi})$ does not possess fixed points on $\partial B_{R}(0)$ for $\tau \in[0,1]$. From the homotopy invariance of degree, it follows that

$$
\operatorname{deg}\left(I-S \circ \Phi, B_{R}(0), 0\right)=\operatorname{deg}\left(I-S \circ \tilde{\Phi}, B_{R}(0), 0\right) .
$$

Since $S \circ \tilde{\Phi}$ has no fixed points in $X$, we see

$$
\operatorname{deg}\left(I-S \circ \tilde{\Phi}, B_{R}(0), 0\right)=0 .
$$

This completes the proof. 
Proof of Theorem 1. By Lemmas 11 and 12, we see

$$
\operatorname{deg}\left(I-S \circ \Phi, B_{R}(0) \backslash B_{r}(0), 0\right)=-1,
$$

which yields a positive solution $v_{\varepsilon}$ of (11) such that

$$
r<\max v_{\varepsilon} \leq R \quad \text { for each } 0<\varepsilon \leq 1 .
$$

Multiplying $v_{\varepsilon} / m\left(v_{\varepsilon}+\varepsilon\right)^{\gamma}$ and $v_{\varepsilon t} /\left(v_{\varepsilon}+\varepsilon\right)^{\gamma}$ with (11) with $v=v_{\varepsilon}$ and integrating over $Q$, respectively, we see that $\left\{v_{\varepsilon}\right\}$ is bounded in $H^{1}(Q)$. Therefore, we may assume that $\left\{v_{\varepsilon}\right\}$ converges to some $v$ weakly in $H^{1}(Q)$ and a.e. in $Q$. It is clear that $\max v \geq r$ and $v \geq 0$ a.e. in $Q$. For every $\varphi \in C_{0}^{\infty}(Q)$, it holds that

$$
\iint_{Q}\left\{\frac{1}{m(1-\gamma)}\left(v_{\varepsilon}+\varepsilon\right)^{1-\gamma} \varphi_{t}-\nabla v_{\varepsilon} \nabla \varphi+h(t) \tilde{f}\left(v_{\varepsilon}\right) \varphi\right\} d x d t=0 .
$$

Letting $\varepsilon \rightarrow 0$, we get

$$
\iint_{Q}\left\{\frac{1}{m(1-\gamma)} v^{1-\gamma} \varphi_{t}-\nabla v \nabla \varphi+h(t) \tilde{f}(v) \varphi\right\} d x d t=0
$$

for every $\varphi \in C_{0}^{\infty}(Q)$. Putting $w_{\varepsilon}=v_{\varepsilon}+\varepsilon$, there is $C_{1}>0$ such that

$$
-\Delta w_{\varepsilon}^{\gamma+1} \leq C_{1}
$$

for all $t \in \mathbb{R}$ and $0<\varepsilon \leq 1$, by (10). Therefore, we have

$$
\int_{\Omega}\left|\nabla w_{\varepsilon}^{\gamma+1}\right|^{2} d x \leq C_{1}|\Omega| R^{\gamma+1} \quad \text { for all } t \in \mathbb{R} \text { and } 0<\varepsilon \leq 1 .
$$

It is immediate that

$$
\iint_{Q}\left|\left(w_{\varepsilon}^{\gamma+1}\right)_{t}\right|^{2} d x d t \leq C_{2} \quad \text { for all } 0<\varepsilon \leq 1
$$

with some $C_{2}>0$. Then it follows that

$$
\begin{aligned}
\int_{\Omega} \mid w_{\varepsilon}^{\gamma+1}(t)-w_{\varepsilon}^{\gamma+1}(s) d x & \leq p\left|\int_{\Omega} \int_{s}^{t}\right| w_{\varepsilon}^{\gamma+1}(\tau)-\left.w_{\varepsilon}^{\gamma+1}(s)\right|^{p-1}\left|\left(w_{\varepsilon}^{\gamma+1}\right)_{t}\right| d x d \tau \mid \\
& \leq p(2 R)^{p-1}\left(2 C_{2}\right)^{1 / 2}|\Omega|^{1 / 2}|t-s|^{1 / 2}
\end{aligned}
$$

for $s, t \in \mathbb{R}$ with $|s-t| \leq T$ and $1 \leq p<+\infty$. This implies the equicontinuity of $\left\{w_{\varepsilon}^{\gamma+1}\right\}_{0<\varepsilon \leq 1}$ from $\mathbb{R}$ into $L^{p}(\Omega)$. According to Ascoli's theorem, $\left\{w_{\varepsilon}^{\gamma+1}\right\}$ converges to $v^{\gamma+1}$ in $L^{p}(\Omega)$ uniformly on every bounded interval of $\mathbb{R}$. Therefore, we obtain $v^{\gamma+1} \in C\left(\mathbb{R} ; L^{p}(\Omega)\right)$ and $v^{\gamma+1}(t+T)=v^{\gamma+1}(t)$ in $L^{p}(\Omega)$ for all $t \in \mathbb{R}$. Setting $u=v^{1 / m}, u$ is our desired weak solution of (1). 
4. Proof of Theorem 2. We can prove Theorem 2 by the same method as in Section 3, except for a priori upper estimates for solutions of (13). Therefore, it suffices to show the following result.

Lemma 13. Under the hypotheses of Theorem 2, there is $M_{0}>0$ such that $\max w \leq M_{0}$ for each solution $w \in C^{2,1}(\bar{\Omega} \times \mathbb{R})$ of (13) with $\Delta w_{t} \in C(\bar{\Omega} \times \mathbb{R})$ and $w>\varepsilon$ in $\Omega \times \mathbb{R}$ and $0<\varepsilon \leq 1$.

Proof. We first get $C_{0}>0$ such that

$$
\int_{0}^{T} w(x, t) d t \leq C_{0}
$$

for each solution $w \in C^{2,1}(\bar{\Omega} \times \mathbb{R})$ of (13) with $\Delta w_{t} \in C(\bar{\Omega} \times \mathbb{R})$ and $w>\varepsilon$ in $\Omega \times \mathbb{R}$ and $0<\varepsilon \leq 1$ in the same way as in the proof of Lemma 3 in [9]. Assuming that the class of such solutions of (13) has no upper bounds, there is a sequence $\left\{w_{n}\right\}$ in $C^{2,1}(\bar{\Omega} \times \mathbb{R})$ of solutions of (13) with $\varepsilon=\varepsilon_{n}\left(0<\varepsilon_{n} \leq 1\right)$ such that $\Delta w_{n t} \in C(\bar{\Omega} \times \mathbb{R}), w_{n}>\varepsilon_{n}$ in $\Omega \times \mathbb{R}$ and

$$
M_{n}=\max w_{n}=w_{n}\left(x_{n}, t_{n}\right) \rightarrow \Omega \quad \text { as } n \rightarrow \infty .
$$

Since

$$
C_{M_{n}} \leq c_{1} M_{n}^{\gamma+1}
$$

for all $n$

with some $c_{1}>0$, where $C_{M_{n}}$ is as in Lemma 1 , it follows that

$$
\int_{t_{n}}^{t_{n}+T} w_{n}\left(x_{n}, t\right) d t \geq \int_{t_{n}}^{t_{n}+1 / c_{1} M_{n}^{\gamma}}\left\{M_{n}-c_{1} M_{n}^{\gamma+1}\left(t-t_{n}\right)\right\} d t=\frac{1}{2 c_{1}} M_{n}^{1-\gamma} \rightarrow \infty
$$

as $n \rightarrow \infty$ from Lemma 1 . This contradicts (26).

\section{Remark 1.}

(i) By the same argument as in Remark 1 in [9], we see that there are no positive solutions of (1) (or (2)) if

$$
\sup _{\rho \neq 0, t \in \mathbb{R}} h(t) \frac{f(\rho)}{\rho^{m}}<\lambda_{1} .
$$

(ii) If

$$
\limsup _{\rho \rightarrow \infty} h(t) \frac{f(\rho)}{\rho^{m}}<\lambda_{1}
$$


uniformly in $t \in \mathbb{R}$ and $f(0) \neq 0$, then there is a nonnegative weak solution of (1).

Indeed, we consider

$$
\begin{cases}v_{t}=m(v+\varepsilon)^{\gamma}(\Delta v+\tau h(t) \tilde{f}(v)) & \text { in } \Omega \times \mathbb{R}, \\ v=0 & \text { on } \partial \Omega \times \mathbb{R}, \\ v(t+T)=v(t) & \text { in } \Omega \times \mathbb{R},\end{cases}
$$

with $\tau \in[0,1]$. By the assumption, there are positive constants $\alpha<\lambda_{1}, C_{0}$ such that

$$
h(t) \tilde{f}(\rho) \leq \alpha \rho+C_{0} \quad \text { for all } \rho \geq 0 \text { and } t \in \mathbb{R} .
$$

Multiplying $1 / m(v+\varepsilon)^{\gamma}$ with (27) and integrating over $(0, T)$ yields

$$
\int_{0}^{T}\left\{\Delta v+\alpha v+C_{0}\right\} d t \geq 0
$$

Putting $V(x)=\int_{0}^{T} v(x, t) d t$, it follows that

$$
\begin{cases}\Delta V+\alpha V+C_{0} T \geq 0 & \text { in } \Omega, \\ V=0 & \text { on } \partial \Omega .\end{cases}
$$

Then $V$ is a subsolution of

$$
\begin{cases}\Delta W+\alpha W+C_{0} T=0 & \text { in } \Omega \\ W=0 & \text { on } \partial \Omega .\end{cases}
$$

It is known that $\max W \leq C_{1}$ for every positive solution $W$ of (29) with some $C_{1}>0$ (see [2]), so $\max V \leq C_{1}$. Therefore, we can obtain a nonnegative weak solution of (27) in the same way as in the proof of Theorem 2.

Acknowledgement. The author is grateful to Professor Claus Dohmen for informing her of several works on porous medium equations.

\section{REFERENCES}

[1] D. Aronson, M. G. Crandall $\mathcal{B}$ L. A. Peletier, Stabilization of solutions of a degenerate nonlinear diffusion problem, Nonlinear Anal. 6 (1982), 1001-1022.

[2] D. G. De Figueiredo, P. L. Lions \& R. D. Nussbaum, A priori estimates and existence of positive solutions of semilinear elliptic equations, J. Math. Pures Appl. 61 (1982), 41-63.

[3] M. Esteban, On periodic solutions of periodic parabolic problems, Trans. Amer. Math. Soc. 93 (1986), 171-189. 
[4] - A remark of the existence of positive periodic solutions of superlinear parabolic problems, Proc. Amer. Math. Soc. 102 (1988), 131-136.

[5] M. FILA, Boundedness of global solutions of nonlinear diffusion equations, J. Differential Equations 98 (1992), 226-240.

[6] B. Gidas, W. M. Ni \& L. Nirenberg, Symmetry and related properties via the maximum principle, Comm. Math. Phys. 68 (1979), 209-243.

[7] Y. GigA, A bound for global solutions of semilinear heat equations, Comm. Math. Phys. 103 (1986), 415-421.

[8] Y. Giga 85 N. MizoguChI, Existence of periodic solutions for equations of evolving curves, (Preprint).

[9] - On the time periodic solutions of the Dirichlet problem for degenerate parabolic equations of non-divergence type, (Preprint).

[10] N. Hirano 8 N. Mizoguchi, Positive unstable periodic solutions for superlinear parabolic equations, Proc. Amer. Math. Soc. 123 (1995), 1487-1495.

[11] O. A. Ladyzhenskaya, V. A. Solonnikov \& N. N. Ural'zeva, Linear and quasilinear equations of parabolic type, Transl. Math. Monograph, no. 23, Amer. Math. Soc., Providence, RI, 1968.

[12] H. A. Levine 8 P. E. SACKS, Some existence and nonexistence theorem for solutions of degenerate parabolic equations, J. Differential Equations 52 (1984), 135-161.

[13] M. NAKAO, Periodic solutions of some nonlinear degenerate parabolic equations, J. Math. Anal. Appl. 104 (1984), 554-467.

[14] Gl Global solutions for some nonlinear parabolic equations with nonmonotonic perturbations, Nonlinear Anal. 10 (1986), 299-314.

[15] W. M. Ni, P. E. SACKS \& J. TAvantzIS, On the asymptotic behavior of solutions of certain quasilinear equations of parabolic type, J. Differential Equations 54 (1984), 97-120.

Department of Mathematics

Tokyo Gakugei University

Koganei

Tokyo, 184 Japan

Received: June 1, 1994. 